\title{
Small Electric and Magnetic Antennas With Cores of a Lossy Dielectric
}

\author{
Janis Galejs
}

\author{
Contribution From Applied Research Laboratory, Sylvania Electronic Systems, a Division of Sylvania \\ Electric Products Inc., 40 Sylvan Road, Waltham 54, Mass.
}

(Received February 18, 1963)

\begin{abstract}
A small loop antenna which is wound on a spherical dielectric core is compared with a cylindrical dielectric filled capacitor. Both antennas provide comparable radiation resistance and reactance for equal antenna volumes. The electric field near the loop is weaker than in the vicinity of the capacitor. This makes the efficiency of the loop less affected by the presence of a lossy dielectric core. For equal efficiencies and volumes of both antennas, the loss tangent of the dielectric in the loop antenna mav be higher than that of the comparable capacitor type by a factor of at least $[\lambda /(2 \pi a)]^{2}$ where $a$ is the antenna radius. Practical design problems are discussed and an approximate method for estimating the losses in loop cores of arbitrary shape is presented.
\end{abstract}

\section{Introduction}

Small electric and magnetic antennas have been discussed by Wheeler [1947, 1958]. He indicates that for a given antenna volume both antenna types exhibit comparable radiation power factors $p$ (or products of bandwidth and efficiency). In the absence of dielectric losses the efficiencies are determined by antenna and tuner losses. However, significant differences between the two antennas occur in case of lossy dielectric cores. Dielectric cores may be required by mechanical considerations under conditions where core materials of negligible losses can be precluded because of the costs involved.

This note will compare a loop antenna wound on a spherical core with a top loaded cylindrical dipole. The above geometries have been selected to facilitate the analysis. Although the discussion is limited to antennas in free space, its conclusions are applicable also to antennas above a conducting ground plane.

The loop antenna is discussed in section 2 using the EMF method. The top loaded dipole is considered in section 3, and the comparison of the two dipole types is shown in section 4 . For a given antenna efficiency it will be seen that dielectric materials of much higher losses can be tolerated in the loop antenna.

\section{Loop Antenna}

\subsection{Spherical Core}

The coil of the loop antenna is wound with $N$ turns on a spherical dielectric core of radius $a$, permittivity $\epsilon_{1}$, conductivity $\sigma_{1}$, and permeability $\mu_{0}$. Free space permittivity $\epsilon_{0}$ and permeability $\mu_{0}$ is assumed for the outside space. The sphere is excited by a surface current density $\boldsymbol{J}_{s}$, the principal component of which is in the $\phi$ direction. After neglecting the $\theta$ component of $J_{s}$, the vector potential can be shown to have a single scalar component $A_{\phi} . \quad A_{\phi}$ is expressible as a summation of Legendre functions, where the coefficients of the individual terms depend on the $J_{s}$ distribution, or on the spread and density of the coil winding. The voltage along the coil is proportional to the line integral of $A_{\phi}$, and the core impedance $Z=V / I$, where $I$ is the coil current. The calculations, which have been summarized in section 5 show that the coil reactance is given by

$$
X=\sqrt{\frac{\mu_{0}}{\epsilon_{0}}} \pi N^{2}\left(k_{2} a\right) f_{1}\left(\theta_{1}\right) .
$$

The radiation resistance is

$$
R_{r}=\sqrt{\frac{\mu_{0}}{\epsilon_{0}}} \frac{\pi}{3} N^{2}\left(k_{2} a\right)^{4} f_{2}\left(\theta_{1}\right),
$$

and the loss resistance due to dielectric losses is

$$
R_{L}=\sqrt{\frac{\mu_{0}}{\epsilon_{0}}} \pi \frac{\epsilon_{1}}{\epsilon_{0}} N^{2}\left(k_{2} a\right)^{3} \tan \delta_{1} f_{3}\left(\theta_{1}\right)
$$

where $\tan \delta_{1}=\sigma_{1} /\left(\omega \epsilon_{1}\right)$.

In the above expressions $k_{2}=\omega \sqrt{\mu_{0} \epsilon_{0}}$ and $f_{i}\left(\theta_{1}\right)$ depends on the distribution of the surface current density $J_{\phi}(\theta)$.

For a coil of a constant pitch with respect to the coil axis $(d z /(a d \phi)=\tan \alpha=$ constant, where $\phi$ is measured along the coil) the surface current density is of the form

$$
J_{\phi}(\theta)=J_{\phi} \sin \theta .
$$


It follows from section 5 that $f_{1}\left(\theta_{1}\right)=f_{2}\left(\theta_{1}\right)=\frac{2}{9}, f_{3}\left(\theta_{1}\right)$ $=\frac{2}{135}$. The length of wire in such a coil is

$$
l=\frac{\pi a}{2 \tan \alpha} .
$$

The parameters of a short coil can be readily evaluated if the surface current density is assumed to be of the form

$$
J_{\phi}(\theta)= \begin{cases}J_{\phi} / \sin \theta & \text { for } \theta_{1}<\theta<\left(\pi-\theta_{1}\right) \\ 0 & \text { elsewhere. }\end{cases}
$$

The pitch of this coil

$$
\frac{d z}{a d \phi}=-\tan \alpha \sin ^{2} \theta
$$

is decreasing toward the poles of the sphere $(\theta=0$, $\pi)$. However, for short coils of $\Delta=\left(\frac{\pi}{2}-\theta_{1}\right)<20^{\circ}$ the winding of the coil (4) does not differ appreciably from the center portion of the winding (6). The calculations of section 5 show that

$$
\begin{gathered}
f_{1}\left(\theta_{1}\right)=S_{1}\left(\theta_{1}\right) f(\Delta) \\
f_{2}\left(\theta_{1}\right)=\frac{1}{2}\left[\frac{\sin \Delta}{\Delta}\right]^{2} f(\Delta) \\
f_{3}\left(\theta_{1}\right)=S_{3}\left(\theta_{1}\right) f(\Delta)
\end{gathered}
$$

where

$$
\begin{gathered}
S_{1}\left(\theta_{1}\right)=\frac{1}{\Delta^{2}} \sum_{n} \frac{1}{n(n+1)}\left[P_{n}\left(\cos \theta_{1}\right)\right]^{2} \\
S_{3}\left(\theta_{1}\right)=\frac{1}{\Delta^{2}} \sum_{n} \frac{1}{n(n+1)(2 n+1)(2 n+3)}\left[P_{n}\left(\cos \theta_{1}\right)\right]^{2} \\
f(\Delta)=4 \Delta^{2}\left[\log \left(\frac{1+\sin \Delta}{1-\sin \Delta}\right)\right]^{-2} \approx 1-\frac{1}{3} \Delta^{2} \text { for } \Delta \leq 1
\end{gathered}
$$

and where $n$ may assume only values of odd positive integers. Plots of $S_{1}\left(\theta_{1}\right)$ and $S_{3}\left(\theta_{1}\right)$ are shown in figure 1. The length of wire in this coil is

$$
l=2 \Delta a / \tan \alpha .
$$

The radiation power factor of the coil (or the product of available bandwidth and efficiency) is computed from (1) and (2) as

$$
p=\frac{R_{r}}{X}=\frac{1}{3}\left(k_{2} a\right)^{3} C_{1}\left(\theta_{1}\right) .
$$

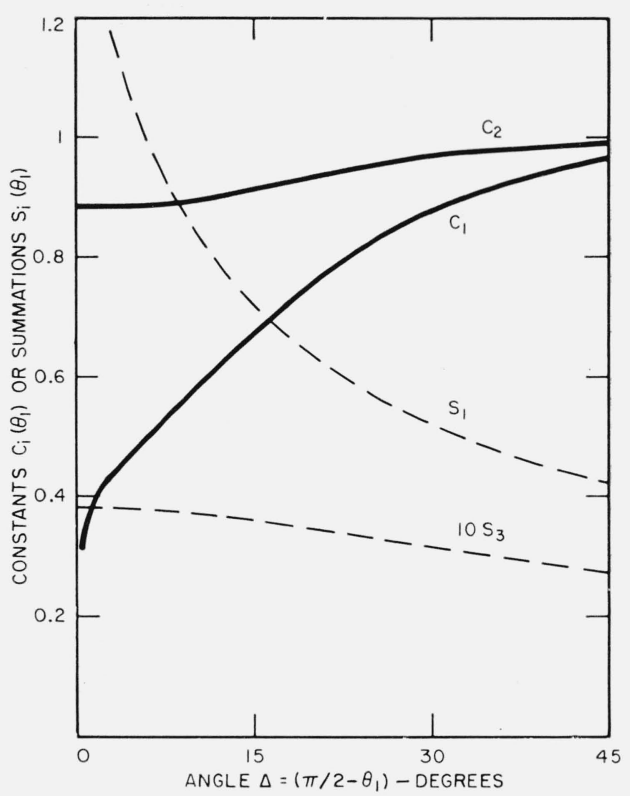

Figure 1. Parameters for determining loop characteristics.

The ratio of radiation to loss resistances is obtained from (2) and (3) as

$$
r=\frac{R_{r}}{R_{L}}=5 \frac{\epsilon_{0}}{\epsilon_{1}} \frac{k_{2} a}{\tan \delta_{1}} C_{2}\left(\theta_{1}\right)
$$

This ratio is approximately equal to antenna efficiency provided $R_{r}<<R_{L}$. The constants $C_{1}\left(\theta_{1}\right)$ and $C_{2}\left(\theta_{1}\right)$ are plotted in figure 1 for $J_{\phi}(\theta)$ of $(6)$, and are equal to unity for $J_{\phi}(\theta)$ of (4).

The above equations for loop antennas are valid only for a uniform current flow through the loop. Phase shifters may be required in larger loops to achieve this condition.

\subsection{Approximations for Different Core Shapes}

The spherically shaped dielectric cores may be encountered only infrequently in engineering applications. Hence, it is desirable to derive approximate expressions for antenna parameters which would also apply to other core shapes. The reactance $X$ and the radiation resistance $R_{r}$ of the coil are in the first approximation unaffected by the presence of the dielectric, and existing free-space formulas may be used for estimating $X$ and $R_{r}$. An approximate calculation of the loss resistance $R_{L}$ may be based on the assumption that the magnetic field inside the core is uniform and equal to the magnetic field which exists in the center of a short coil of radius $a$.

In a cylindrical coordinate system where the $z$ axis is alined with the axis of the loop, the magnetic field component $H_{z}$ is given by (7.10.8) of Smythe $[1950]$ as

$$
H_{z}=\frac{I N}{2 a} \text {. }
$$


The associated electric field component $E_{\phi}$ is computed from Faraday's law

$$
\int_{0}^{2 \pi} E_{\phi} \rho d \phi=-\frac{d}{d t} \int_{0}^{2 \pi} \int_{0}^{\rho} B_{z} \rho d \rho d \phi
$$

as

$$
\left|E_{\phi}\right|=\frac{1}{2} \omega \mu_{0} \rho H_{z}
$$

The power loss in the dielectric is given by the equation

$$
P_{L}=\sigma_{1} \int\left|E_{\phi}\right|^{2} \rho d \rho d \phi d z
$$

and the loss resistance $R_{L}$ becomes

$$
R_{L}=\frac{P_{L}}{I^{2}}=\sigma_{1} \frac{\pi}{8}\left[\frac{\omega \mu_{0} N}{a}\right]^{2} \int_{0}^{\rho_{1}} d \rho \rho^{3} \int_{z_{1}(\rho)}^{z_{2}(\rho)} d z
$$

where the limits of integration depend on the geometry of the core.

For a spherical dielectric core, (21) will be compared with the more accurate expression (3). For a sphere where $z_{1,2}(\rho)= \pm \sqrt{a^{2}-\rho^{2}}$ the integrals of $(21)$ can be readily evaluated to give

$$
R_{L}=\frac{\pi \epsilon_{1}}{30 \epsilon_{0}} \sqrt{\frac{\mu_{0}}{\epsilon_{0}}} N^{2}\left(\beta_{2} a\right)^{3} \tan \delta_{1} .
$$

The resistance $R_{L}$ of $(22)$ is approximately 10 percent less than $R_{L}$ for $J_{\phi}(\theta)$ of $(6)$ with short coils $\left(\Delta<20^{\circ}\right)$, but is approximately twice the $R_{L}$ for $J_{\phi}(\theta)$ of (4), which is achieved by a coil covering all of the sphere. The crude approximation of the magnetic field in (17) is therefore useful for obtaining an estimate of $R_{L}$ for short coils and an upper bound of $R_{L}$ for wide coils in a spherical geometry. The same general conclusion may be expected to apply to hemispherical, ellipsoidal, or other related core geometries.

\section{Dipole Antenna}

The dielectric loaded dipole antenna has been treated in the biconical geometry of figure 2a by Galejs [1962b]. However, for purposes of illustrating the differences with loop antennas the simple cylindrical capacitor shape of figure $2 \mathrm{~b}$ is sufficient. In the absence of dielectric loading $\left(\epsilon_{1}=\epsilon_{0}\right)$ its capacity is

$$
C=k_{a} \frac{\epsilon_{0} \pi b^{2}}{h}
$$

where $b=a \sin \Delta$ and $h=2 a \cos \Delta$. The shape factor of the capacitor $k_{a}$ is given by Vilbig [1960] as

$$
k_{a}=1+\frac{h}{\pi b}\left[\log \frac{16 \pi b}{h}+1\right] \text {. }
$$

With dielectric loading $\left(\epsilon_{1} \neq \epsilon_{0}\right)$ the capacity is approximated by

$$
C=\left(k_{a}-1+\frac{\epsilon_{1}}{\epsilon_{0}}\right) \frac{\epsilon_{0} \pi b^{2}}{h} .
$$

The radiation conductance is independent of dielectric loading [Wheeler, 1947; Galejs, 1962a] and is given by

$$
G_{r}=\frac{\pi}{6} \sqrt{\frac{\epsilon_{0}}{\mu_{0}}} k_{a}^{2}\left(k_{2} b\right)^{4}
$$

The power loss in the dielectric may be computed as

$$
P_{L}=\sigma_{1} \int|E|^{2} d v=\frac{\sigma_{1}}{\epsilon_{1}} C_{i} V^{2}
$$

where $V$ is the voltage applied to the capacitor and the capacity $C_{i}$ of the antenna due to the energy storage inside the dielectric is

$$
C_{i}=\frac{\epsilon_{1} \pi b^{2}}{h} .
$$

These power losses may be accounted for by a loss conductance

$$
G_{L}=\frac{P_{L}}{V^{2}}=\frac{\sigma_{1} \pi b^{2}}{h} .
$$

The radiation power factor of the dipole antenna is computed from (25) and (26) as

$$
p=\frac{G_{r}}{\omega C}=\frac{1}{6} \frac{k_{a}^{2}}{k_{a}-1+\frac{\epsilon_{1}}{\epsilon_{0}}} \frac{h}{b}\left(k_{2} b\right)^{3} .
$$

The ratio of radiation conductance $G_{r}$ to loss conductance $G_{L}$ is obtained from (26) and (29) as

$$
r=\frac{G_{r}}{G_{L}}=\frac{1}{6} \frac{\epsilon_{0}}{6} \frac{k_{a}^{2}}{\tan \delta_{1}} \frac{h}{b}\left(k_{2} b\right)^{3} .
$$

This ratio is the same as the ratio of radiation resistance $R_{r}$ to loss resistance $R_{L}$, because $R_{i}=$ $G_{i}(\omega C)^{-2}$ for $i=r$ or $L$.
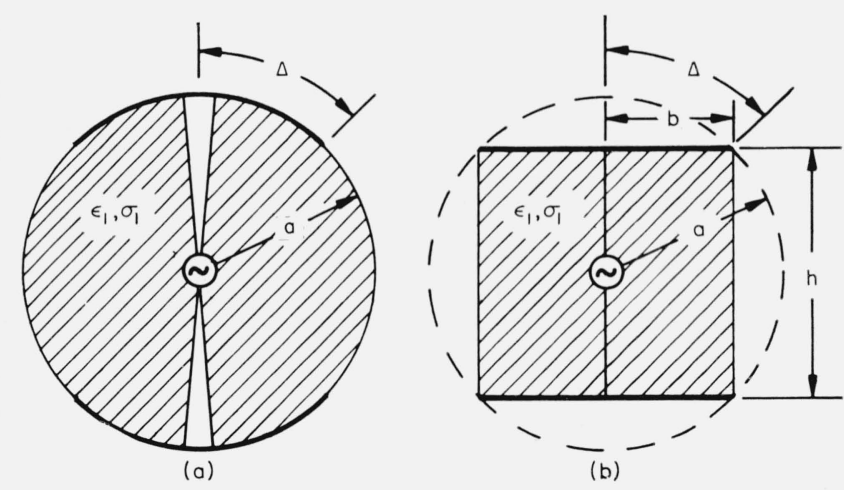

Figure 2. Biconical and cylindrical dipole antennas. 


\section{Comparison of the Two Antenna Types}

The loop antenna of a spherical dielectric core of radius $a$ will be compared with a cylindrical parallel plate dipole antenna which fits in a sphere of same radius as indicated in figure $2 \mathrm{~b}$. The dielectric constant is the same for both antennas, but not the dielectric loss tangents. The parameters of the two antennas are distinguished by subscripts $m$ (magnetic) and $e$ (electric).

The radiation power factors of the two antennas are related from (15) and (30) as

$$
\frac{p_{m}}{p_{e}}=\frac{2\left(k_{a}-1+\frac{\epsilon_{1}}{\epsilon_{0}}\right) C_{1}\left(\theta_{1}\right) a^{3}}{k_{a}^{2} b^{2} h} .
$$

For $\Delta=45^{\circ}$ and $\epsilon_{1} / \epsilon_{0}=3, p_{m} / p_{e}=1.2$ and 1.16 for $J_{\phi}(\theta)$ of (4) and $(6) ; p_{m}$ is about the same as $p_{e}$. However, the ratio of $\left(p_{m} / p_{e}\right)$ is decreased by decreasing $\epsilon_{1} / \epsilon_{0}$.

The ratio of the radiation to loss resistance ratio of the two antennas follows from (16) and (31) as

$$
\frac{r_{m}}{r_{e}}=\frac{30 C_{2}\left(\theta_{1}\right) a^{3}}{k_{a}^{2} h b^{2}} \frac{\tan \delta_{1 e}}{\tan \delta_{1 m}\left(k_{2} a\right)^{2}}=K_{1} \frac{\tan \delta_{1 e}}{\tan \delta_{1 m}\left(k_{2} a\right)^{2}} .
$$

For $\Delta=45^{\circ}, K_{1}=3.15$ and 3.1 for $J_{\phi}(\theta)$ of (4) and (6). For equal efficiencies $\left(r_{m}=r_{e}\right)$ and $k_{2} a<<1$, the magnetic antenna may have much higher losses (tan $\delta_{1 m}>>\tan \delta_{1 e}$ ). Assuming $k_{2} a=0.1$, $\tan \delta_{1 m}$ may exceed $300 \tan \delta_{1 e}$. The requirements imposed on the insulating qualities of the dielectric are considerably less stringent in a loop type antenna. This can be very significant in antenna design problems where the cost of a high quality dielectric may become prohibitive. This difference between the two antenna types may be explained qualitatively by examining the behavior of the electric field in the vicinity of the antenna. For equal amounts of radiated power both antennas will exhibit equal radiation resistances $R_{r}$ and approximately the same far fields. However, the electric field in the near zone of the electric dipole is $\left(k_{2} r\right)^{-1}$ times higher than with the magnetic dipole ( $r=$ distance to the center of the dipole). The losses which are proportional to $\mid E^{2}$ will be $\left(k_{2} a\right)^{-2}$ higher, which explains the functional form of (33).

In many instances, bandwidth considerations restrict the efficiency of small sized antennas. For $k_{2} a=0.1$ and $\Delta=45^{\circ}, p_{m}=3.2 \times 10^{-4}$ from (15). For a 1 percent relative bandwidth, the efficiency $r_{m}$ of (16) should not exceed $3.2 \times 10^{-2}$. This efficiency can be achieved with $\tan \delta_{1} \approx 5$ in absence of any further antenna losses. As long as materials of tan $\delta_{1} \approx 1$ are available, there should be little concern about the ohmic wire losses or losses in the coupling network.

Equation (33) with $K_{1}=1$ applies also to electric and magnetic dipoles which are surrounded by a spherical shell of lossy dielectric, provided that the shell is thin compared to the skin depth and $k_{2} a$ $<<1$, where $\mathrm{a}=$ mean radius of the shell. This follows* from a formulation which is similar to that of Raemer [1962], and which yields simple closed form solutions for $k_{2} a<<1$.

It will be further shown that the loop antenna can radiate power which is comparable with the power radiated by the electric dipole for a given antenna. current. The number of turns $N$ of the loop antenna which is required for achieving equal radiation resistances $\left(R_{r e}=R_{r m}\right)$ is computed from (2), (25), and (26) as

$$
N=\frac{1}{\pi \sqrt{2 f_{2}\left(\theta_{1}\right)}} \frac{k_{a}}{k_{a}-1+\frac{\epsilon_{1}}{\epsilon_{0}}} \frac{h}{a} \frac{1}{k_{2} a}=K_{2} \frac{1}{k_{2} a} .
$$

For $\Delta=45^{\circ}$ and $\epsilon_{1} / \epsilon_{0}=3, K_{2}=0.44$ and 0.33 for $J_{\phi}(\theta)$ of (4) and (6). For $k_{2} a=0.1$ only 3 to 5 turns are required for the coil. However, these turns should be spread out over a sizable surface area of the sphere $\left(\Delta>45^{\circ}\right)$. A close packing of the turns. decreases $\Delta, C_{1}\left(\theta_{1}\right)$, and $p_{m}$ of $(15)$.

A loop of widely spaced turns will have an appreciable $\theta$ component $J_{\theta}$ of the surface current density $J_{s}$, which has been neglected thus far. This surface current density $J_{\theta}$ causes an electric field and also an electric dipole moment in the direction of the loop axis. The dielectric losses which are caused by this. axial electric field require further consideration. With $p_{m} \approx p_{e}$ and $N$ of (34) it follows that $R_{r e}=R_{r m}$. and $X_{e} \approx\left|X_{m}\right|$. For equal currents the voltage across the capacitor $V_{e}$ will be equal to the voltage between the terminals of the coil $V_{m}$, which may, at first sight, indicate power losses $P_{L m}$ comparable to the losses. $P_{L e}$. However, in the case of coil, the electric field is. not uniform over the volume of the dielectric and it is. strongest near the wire surface. The losses $P_{L m}$ may be kept to a tolerable level by insulating the wires. with high quality dielectric of sufficient thickness. The losses due to the electric field between two insulated wires in a lossy dielectric will be less than in a coaxial cable of the same insulation, and the proper design of the latter is a simple problem.

The previous discussion has dealt with the free space performance of spherical core antennas. The above conclusions will also apply to antennas of hemispherical or similarly shaped cores above a. highly conducting half-space. The efficiency of a small lossy-core loop antenna will be in most cases. restricted by bandwidth considerations.

The previous comparison is clearly in favor of the loop antenna. This is an obvious consequence of differences in the $E$ field dependence between electric and magnetic dipoles. However, these results have not been pointed out in the literature before and may be of considerable interest in practical design problems.

\section{Appendix. Analysis of the Loop Antenna}

\subsection{Vector Potential}

The loop antenna is wound on a dielectric sphere of radius $a$ and of dielectric constant $\epsilon_{1}$, magnetic permeability $\mu_{0}$, and conductivity $\sigma_{1}$. The outer

\footnotetext{
*Private communication by Dr. R. V. Row of this laboratory.
} 
medium is characterized by $\epsilon_{2}, \mu_{2}$, and $\sigma_{2}$. The parameters $\epsilon_{2}$ and $\sigma_{2}$ are kept arbitrary in this section in order to afford comparisons with known results for loops in infinite lossy media [Wait, 1952; Kraichman, 1962]. The sphere is excited by a surface current of density $\mathbf{J}_{s}=J_{\phi} \mathbf{i}_{\phi}+J_{\theta} \mathbf{i}_{\theta}$. When ignoring the $\theta$ component of $\mathbf{J}_{s}$ the vector potential $\mathbf{A}$ will have a single component $A_{\phi}$, which may be computed following sections 7.13 and 14.14 of Smythe [1950]. For a suppressed $e^{j \omega t}$ time variation of the fields and for $r<a, A_{\phi 1}$ is given by

$$
\begin{aligned}
A_{\phi 1}=-\sum_{n} \frac{\mu_{0} C_{n}}{k_{1} a} & {\left[\frac{S_{n}^{\prime}\left(k_{1} a\right)}{S_{n}\left(k_{1} a\right)}\right.} \\
& \left.-\frac{k_{2}}{k_{1}} \frac{R_{n}^{\prime}\left(k_{2} a\right)}{R_{n}\left(k_{2} a\right)}\right]^{-1} P_{n}^{1}(\cos \theta)\left(\frac{r}{a}\right)^{n}
\end{aligned}
$$

where

$$
\begin{gathered}
C_{n}=-\frac{(2 n+1) a}{2 n(n+1)} \int_{0}^{\pi} J_{\phi}(\theta) P_{n}^{1}(\cos \theta) \sin \theta d \theta \\
k_{i}=\beta_{i}-j \alpha_{i}=\sqrt{\omega^{2} \mu_{0} \epsilon_{i}-j \omega \mu_{0} \sigma_{i}} \\
R_{n}(x)=\sqrt{x} H_{n+1 / 2}^{(2)}(x) \\
S_{n}(x)=\sqrt{x} J_{n+1 / 2}(x)
\end{gathered}
$$

$H_{n}^{(2)}(x)$ is the Hankel function of the second kind of order $n$, and $J_{n}(x)$ is the Bessel function of order $n$. The prime denotes differentiation with respect to the argument of the respective function. The associated Legendre function $P_{n}^{1}(\cos \theta)$ is defined by

$$
P_{n}^{1}(\cos \theta)=-\frac{d}{d \theta} P_{n}(\cos \theta)
$$

where $P_{n}(\cos \theta)$ is the Legendre polynomial of order $n$. The summation covers only odd integers $(n=2 m+1, \quad m=$ positive integer or 0$)$, and it is stipulated that $k_{1} a \mid \ll 1$. After using the small argument approximations of the $H_{n}^{(2)}(x)$ and $J_{n}(x)$ functions, it follows that

$$
\begin{array}{r}
\frac{1}{k_{1} a}\left[\frac{S_{n}^{\prime}\left(k_{1} a\right)}{S_{n}\left(k_{1} a\right)}-\frac{k_{2}}{k_{1}} \frac{R_{n}^{\prime}\left(k_{2} a\right)}{R_{n}\left(k_{2} a\right)}\right]^{-1} \\
\approx \frac{1}{2 n+1}\left[1+\frac{\left(k_{1} a\right)^{2}}{(2 n+1)(2 n+3)}+\frac{\left(k_{2} a\right)^{2}}{(2 n+1)(2 n-1)}\right. \\
\left.\quad-\frac{j\left(k_{2} a\right)^{3} \delta_{1 n}}{3}\right]=\frac{D_{n}}{2 n+1}
\end{array}
$$

where the Kronecker Delta is defined by

$$
\delta_{1 n}=\left\{\begin{array}{l}
1 \text { for } n=1 \\
0 \text { for } n \neq 1
\end{array}\right.
$$

The coefficients $C_{n}$ depend on the distribution of $J_{\phi}(\theta)$ over the sphere surface.

\subsection{Surface Current of Density Proportional to $\sin \theta$}

A surface current density

$$
J_{\phi}(\theta)=J_{\phi} \sin \theta
$$

gives particularly simple results for the impedance. Substitution of (43) in (36) results in

$$
C_{n}=-J_{\phi} a \delta_{1 n}
$$

with $\delta_{1 n}$ defined by (42). Along the winding of the coil

$$
\frac{d \phi}{d \theta}=-\sin \theta / \tan \alpha
$$

where $\tan \alpha$ is the pitch of the coil $d z /(a d \phi)$. The wire current may be computed as

$$
I=\int_{90^{\circ}+\pi(d \theta / d \phi)}^{90^{\circ}-\pi(d \theta / d \phi)} a J_{\phi}(\theta) d \theta \approx 2 \pi a J_{\phi} \tan \alpha .
$$

The number of turns $N$ is obtained from

$$
2 \pi N=\int_{\phi_{1}}^{\phi_{2}} d \phi=\frac{1}{\tan \alpha} \int_{0}^{\pi} \sin \theta d \theta=\frac{2}{\tan \alpha}
$$

as

$$
N=(\pi \tan \alpha)^{-1} .
$$

The length of wire in such a coil is

$$
l=\int_{\phi_{1}}^{\phi_{2}} a \sin \theta d \phi=\frac{a}{\tan \alpha} \int_{0}^{\pi} \sin ^{2} \theta d \theta=\frac{\pi a}{2 \tan \alpha} .
$$

Applying (41), (44), (46), and (48), $A_{\phi 1}$ becomes

$$
A_{\phi 1}=\frac{\mu_{0} N I}{6}\left(\frac{r}{a}\right) D_{1} \sin \theta .
$$

The voltage impressed on the coil is

$$
\begin{aligned}
V=-\int \mathbf{E}_{\phi} \cdot \mathbf{d} \mathbf{l}=j \omega a \int_{\phi_{1}}^{\phi 2} & A_{\phi} \sin \theta d \phi \\
& =j \omega a N \pi \int_{0}^{\pi} A_{\phi} \sin ^{2} \theta d \theta
\end{aligned}
$$

where $r=a$. After evaluating the $\theta$ integral, the impedance of the coil is computed as

$$
Z=\frac{V}{I}=\frac{2 \pi}{9} j \omega \mu_{0} a N^{2} D_{1}
$$

The real part of $D_{n}$ of (41) when substituted in (52) gives the coil reactance. Thus

$$
X=\frac{2 \pi}{9} \omega \mu_{0} a N^{2}=\sqrt{\frac{\mu_{0}}{\epsilon_{2}}} \frac{2 \pi}{9} N^{2} \beta_{2} a .
$$


The imaginary part of terms of $D_{n}$ proportional to powers of $\beta_{2}$ gives the radiation resistance. For a lossless medium $2\left(\sigma_{2}=0\right)$ the radiation resistance is

$$
R_{r}=\sqrt{\frac{\mu_{0}}{\epsilon_{2}}} \frac{2 \pi}{27} N^{2}\left(\beta_{2} a\right)^{4} .
$$

The imaginary part of terms of $D_{n}$ proportional to $\alpha_{1}$ gives the loss resistance $R_{L}$. For a lossless medium $2\left(\sigma_{2}=0\right) R_{L}$ becomes

$$
R_{L}=\sqrt{\frac{\mu_{0}}{\epsilon_{2}}} \frac{2 \pi}{135} \frac{\epsilon_{1}}{\epsilon_{2}} N^{2}\left(\beta_{2} a\right)^{3} \tan \delta_{1} .
$$

Equations (53) and (54) agree with (26) and (27) of Wheeler [1958], respectively.

\subsection{Surface Current of Density Proportional to $(\sin \theta)^{-1}$}

The $\theta$ integrals involved in the impedance calculations may be evaluated also for a nonuniform current density

$$
J_{\phi}(\theta)= \begin{cases}J_{\phi} / \sin \theta & \text { for } \theta_{1} \leq \theta \leq\left(\pi-\theta_{1}\right) \\ 0 & \text { elsewhere }\end{cases}
$$

with $\theta_{1}=\frac{\pi}{2}-\Delta$. Substituting (56) $C_{n}$ becomes

$$
C_{n}=-\frac{(2 n+1) a J_{\phi}}{2 n(n+1)} \int_{\theta_{1}}^{\theta_{2}} P_{n}^{1}(\cos \theta) d \theta
$$

After substituting (40), integration gives

$$
C_{n}=-\frac{(2 n+1) a J_{\phi}}{n(n+1)} P_{n}\left(\cos \theta_{1}\right) .
$$

Along the winding of the coil

$$
\frac{d \phi}{d \theta}=(\tan \alpha \sin \theta)^{-1}
$$

where $\tan \alpha$ is the pitch of the coil $d z /(a d \phi)$ at $\theta=\pi / 2$. The wire current $I$ is related to the surface current density $J_{\phi}$ by (46). The number of turns is obtained from

$$
\begin{aligned}
2 \pi N=\int_{\phi_{1}}^{\phi_{2}} d \phi=\frac{1}{\tan \alpha} \int_{\theta_{1}}^{\theta_{2}} \frac{d \theta}{\sin \theta} & \\
& =\frac{1}{\tan \alpha} \log \frac{1+\sin \Delta}{1-\sin \Delta} .
\end{aligned}
$$

The length of wire in such a coil is

$$
l=\int_{\phi_{1}}^{\phi_{2}} a \sin \theta d \phi=\frac{a}{\tan \alpha} \int_{\theta_{1}}^{\theta_{2}} d \theta=\frac{2 \Delta a}{\tan \alpha} .
$$

In the limit of $\Delta=\pi / 2, N=\infty$, but $l$ is finite and is twice the $l$ of (49). [Wheeler, 1958.]

Applying (41), (46) and (58), $A_{\phi 1}$ becomes

$$
A_{\phi 1}=\frac{\mu_{0} I}{2 \pi \tan \alpha} \sum_{n} \frac{D_{n}}{n(n+1)}\left(\frac{r}{a}\right)^{n} P_{n}\left(\cos \theta_{1}\right) P_{n}^{1}(\cos \theta) .
$$

The voltage impressed on the coil is

$$
\begin{aligned}
V=-\int \mathbf{E}_{\phi} \cdot d \mathbf{l}=j \omega a \int_{\phi_{1}\left(\theta_{1}\right)}^{\phi_{2}\left(\theta_{2}\right)} A_{\phi} & \sin \theta d \phi \\
& =\frac{j \omega a}{\tan \alpha} \int_{\theta_{1}}^{\theta_{2}} A_{\phi} d \theta
\end{aligned}
$$

where $r=a$. The $\theta$ integral may be evaluated as in (57) and the impedance of the coil is computed as

$$
Z=\frac{V}{I}=\frac{j \omega \mu_{0} a \sin ^{2} \theta_{1}}{\pi \tan ^{2} \alpha} \sum_{n} \frac{D_{n}}{n(n+1)}\left[P_{n}\left(\cos \theta_{1}\right)\right]^{2}
$$

where $n=2 m+1$, with $m$ equal to zero or positive integers. The reactance $X$, radiation resistance $R_{r}$ and the loss resistance $R_{L}$ are obtained after substituting (41) as

$$
\begin{gathered}
X=\omega \mu_{0} a \pi N^{2} S_{1}\left(\theta_{1}\right) f(\Delta) \\
=\sqrt{\frac{\mu_{0}}{\epsilon_{2}}} \pi N^{2} \beta_{2} a S_{1}\left(\theta_{1}\right) f(\Delta) \\
R_{r}=\sqrt{\frac{\mu_{0}}{\epsilon_{2}}} \frac{\pi}{6} N^{2}\left(\beta_{2} a\right)^{4}\left[\frac{\cos \theta_{1}}{\Delta}\right]^{2} f(\Delta) \\
R_{L}=2 \pi \omega \mu_{0} N^{2} \alpha_{1} \beta_{1} a^{3} S_{3}\left(\theta_{1}\right) f(\Delta) \\
=\sqrt{\frac{\mu_{0}}{\epsilon_{2}}} \frac{\epsilon_{1}}{\epsilon_{2}} \pi N^{2}\left(\beta_{2} a\right)^{3} \tan \delta_{1} S_{3}\left(\theta_{1}\right) f(\Delta)
\end{gathered}
$$

where $S_{1}\left(\theta_{1}\right), S_{3}\left(\theta_{1}\right)$ and $f(\Delta)$ are defined by (11) to (13). The same expression for $R_{L}$ is obtained from the power loss $P_{L}$ in the volume of the sphere as $R_{L}=P_{L} / I^{2} . \quad P_{L}$ is computed as

$$
P_{L}=\sigma \int_{0}^{2 \pi} d \phi \int_{0}^{\pi} d \theta \int_{0}^{a} d r \sin \theta r^{2}\left|\omega A_{\phi 1}\right|^{2}
$$

where $A_{\phi 1}$ is given by (62). $R_{L}$ of (67) may be compared with the loss resistance $R_{L_{\infty}}$ of the same coil in an infinite lossy medium. $R_{L_{\infty}}$ is obtained similarly as (67) but by setting $k_{1}=k_{2}$ in (41). For $\Delta<<1$ this gives

$$
R_{L_{\infty}}=\frac{4}{3} \omega \mu_{0} N^{2} \alpha_{1} \beta_{1} a^{3}=\frac{2}{3} \sqrt{\frac{\mu_{0}}{\epsilon_{2}}} \frac{\epsilon_{1}}{\epsilon_{2}} N^{2}\left(\beta_{2} a\right)^{3} \tan \delta_{1}
$$

which is approximately $5 R_{L}$. In a highly conducting medium displacement currents are negligible, $\alpha_{1}=\beta_{1}$ and (69) is reduced to a known result [Kraichman, 1962]. The difference between (69) and (67) is equal to the loss resistance $R_{L 0}$ of a coil within a lossless radome. It follows that

$$
R_{L 0}=1.096 \omega \mu_{0} N^{2} \alpha_{2} \beta_{2} a^{3} .
$$


This is approximately 5 percent more than the loss resistance of an infinitesimal magnetic dipole which is assumed to have an area equal to the cross section $\pi a^{2}$ of region 1 [Wait, 1952].

\section{References}

Galejs, J. (Sept.-Oct. 1962a), Dielectric loading of electric dipole antennas, J. Research NBS 66D (Radio Prop.) No. $5,557-562$.

Galejs, J. (Dec. 1962b), Capacitor type biconical antennas, Res. Rpt., No. 319, Applied Research Laboratory, Sylvania Electronic Systems, Waltham, Mass.

Kraichman, M. B. (July-Aug. 1962), Impedance of a circular loop in an infinite conducting medium, J. Research NBS 66D (Radio Prop.) No. 4, 499-503, see equation (7).
Raemer, H. R., (1962), Radiation from linear electric and magnetic antennas surrounded by a spherical plasma shell, IRE Trans. on Ant. and Prop. AP-10, No. 1, 69-78.

Smythe, W. R. (1950), Static and dynamic electricity (McGraw-Hill Book Co., Inc., New York, N.Y.).

Vilbig, F. (1960), Lehrbuch der Hochfrequenztechnik 1, Akademische Verlagsges, Frankfurt am Main, 147.

Wait, James R. (Oct. 1952), The magnetic dipole antenna immersed in a conducting medium, Proc. IRE 40, No. 10, 1244-1245.

Wheeler, H. A. (Dec. 1947), Fundamental limitations of small antennas, Proc. IRE 35, No. 12, 1479-1484.

Wheeler, H. A. (Sept. 1958), The spherical coil as an inductor shield or antenna, Proc. IRE 46, No. 9, 1595-1602.

(Paper 67D4-279) 